\title{
Design of Constant Gain Dissipative Controllers for Eigensystem Assignment in Passive Systems
}

\author{
Peiman G. Maghami ${ }^{\dagger}$ \\ NASA Langley Research Center \\ Sandeep Gupta \\ Hughes Space and Communications Company
}

\begin{abstract}
Partial eigensystem assignment with output feedback can lead to an unstable closed-loop system. However, output feedback with passive linear time-invariant systems, such as flexible space structures, is guaranteed to be stable if the controller is dissipative. This paper presents a novel approach for synthesis of dissipative output feedback gain matrices for assigning a selected number of closed-loop poles. Dissipativity of a gain matrix is known to be equivalent to positive semidefiniteness of the symmetric part of the matrix. A sequential procedure is presented to assign one self-conjugate pair of closed-loop eigenvalues at each step using dissipative output feedback gain matrices, while ensuring that the eigenvalues assigned in the previous steps are not disturbed. The problem of assigning one closed-loop pair is reduced to a constrained solution of a system of quadratic equations, and necessary and sufficient conditions for the existence of a solution are presented. A minimax approach is presented for determining parameters which satisfy these conditions. This method can assign as many closed-loop system poles as the number of control inputs. A numerical example of damping enhancement for a flexible structure is presented to demonstrate the approach.
\end{abstract}

\footnotetext{
$\dagger$ Senior Research Engineer, MS 161, Guidance and Control Branch, Flight Dynamics and Control Division, NASA Langley Research Center, Hampton, VA 23681-0001.

* Senior Staff Engineer, Hughes Space and Communications Company, Los Angeles, CA 90009.
} 


\section{Introduction}

Pole placement has been studied extensively in the literature to shape transient response of the closed-system. Many techniques are available for eigensystem assignment with full state feedback. These techniques exploit the freedom beyond pole placement for multivariable systems, identified in Ref. 1, for other objectives such as enhancing robustness of the pole placement and minimizing the norm of the feedback gain needed to accomplish the placement ${ }^{2,3}$. However, full state feedback is not feasible for many control applications such as control of flexible structures. The dynamics of flexible structures is described by infinite dimensional equations and is usually approximated by large second-order discrete models; therefore, the measurement of the full state is not feasible. Output feedback pole placement is the practical choice for such systems. However, much of the literature for output feedback eigensystem assignment deals with relatively low order systems, where the number of states is less than the sum of inputs and outputs ${ }^{4,5,6}$, and therefore is not applicable for problems like control of flexible structures. Conditions on the closed-loop eigenvectors for the existence of output feedback gain matrices to accomplish the desired eigenvalue placement are discussed in Ref. 7. References 8 and 9 present techniques for synthesis of output feedback gains, which assign a self-conjugate subset of the closed-loop eigenvalues. However, a major drawback of using output feedback for eigensystem assignment of large order systems is that while assigning a self-conjugate set of eigenvalues, the feedback may cause other closed-loop poles of the system to become unstable ${ }^{10}$. Therefore, it is desirable to develop techniques for output feedback gain matrices which assign dominant modes of the system while ensuring that the overall system is not destabilized. This problem is addressed in the current paper for strictly passive linear timeinvariant systems in descriptor form, which are used in the characterization of the dynamics of flexible space structures.

Robust control of flexible space structures is difficult due to its dynamic characteristics, namely, numerous closely-spaced, low frequency modes with little inherent damping ${ }^{11}$. Moreover, the parameters associated with flexible structure models such as modal frequencies, 
damping and mode shapes often cannot be identified accurately. Feedback control is further complicated by instabilities induced by observation and control spillovers ${ }^{11}$. However, with collocated and compatible actuators and rate sensors, the system dynamics are passive, irrespective of unmodelled dynamics and parametric uncertainty ${ }^{12,13,14}$. Moreover, by the passivity theorem ${ }^{15}$, a strictly passive controller guarantees robust stability of the closed-loop system. For constant gain feedback, strict passivity requires that the symmetric part of the constant gain matrix be positive definite. Note that symmetry of the gain matrix is not necessary for strict passivity. Positive definiteness of the symmetric part of the output feedback gain matrix is necessary and sufficient for strict passivity. Similarly, when the plant is strictly passive, a passive controller ensures robust closed-loop stability. Positive semidefiniteness of the symmetric part of the gain matrix ensures passivity of a constant gain feedback controller. Thus, a strictly passive system is guaranteed to be robustly stable with constant gain output feedback if the symmetric part of the gain matrix is positive semidefinite.

Thus, the robust eigensystem assignment problem for strictly passive systems is to construct output feedback gain matrices whose symmetric part is positive semidefinite. Preliminary approaches to these problems are described in Refs. 16 and 17. Reference 17 presents a sequential procedure through which a pair of self-conjugate eigenvalues is assigned to desired locations at every step with the aid of generalized Schur decompositions and orthogonal state transformations. This procedure is repeated until up to $m$ self-conjugate closed-loop eigenvalues have been assigned, where $m$ is the number of control inputs to the system. At each step the output feedback gains are designed such that the previously placed eigenvalues remain unchanged. This approach reduces the problem of assigning one self-conjugate pair of closedloop eigenvalues to constrained solution of a set of quadratic equations. In this paper, necessary and sufficient conditions for the existence of a solution to these quadratic equations are presented in this paper for two cases, namely, the nonsymmetric gain matrices and the symmetric gain matrices. Furthermore, a minimax approach is described for obtaining parameters which satisfy the conditions for pole placement. Once parameters satisfying these conditions for pole placement have been determined, the approach to evaluate output feedback gain matrices follows. These 
results are first developed for assigning dominant poles of passive LTI systems in descriptor form, in particular, for damping enhancement of flexible space structures with rate feedback. Then, these techniques are extended to pole placement with both position and rate feedback for second-order models of mechanical systems, while ensuring stability of the overall closed-loop system. The conditions guaranteeing stability in this case are that the symmetric part of the rate gain matrix is positive semidefinite, and the position gain matrix is symmetric and positive semidefinite. Corresponding quadratic equations and conditions for existence of a solution for eigenvalue assignment using both rate and position feedback are presented. Finally, a numerical example of damping enhancement in the dominant modes of a structural testbed at NASA Langley (CEM phase II) is presented to demonstrate the application of this approach.

\section{Passive LTI Systems}

Systems that do not generate energy internally are referred to as passive systems. Mathematically, input-output systems are defined to be passive if $\langle y, u\rangle \geq \beta$, where $\langle\cdot, \cdot\rangle$ denotes the inner product of the input-output vector spaces, $u$ is the input to the system, $y$ is the output of the system, and $\beta$ is a parameter corresponding to the initial conditions of the system $^{15}$. First, consider a constant gain system, $y=G u$, where $G$ is the constant gain matrix. Note that $y^{T} u=u^{T} G u=u^{T}(\operatorname{sym}\{G\}) u$, where $\operatorname{sym}\{G\}$ denotes the symmetric part of $G$, defined as $\operatorname{sym}\{G\}=\frac{1}{2}\left(G+G^{T}\right)$. Now, $\langle y, u\rangle \geq 0$ (for passivity with $\beta=0$ ) if and only if $\operatorname{sym}\{G\} \geq 0$, where " $\geq 0$ " denotes positive semidefiniteness of the matrix on the left-hand side of the inequality. Thus, it follows directly from the definition that positive semidefiniteness of the symmetric part of the gain matrix, $G$, is equivalent to passivity of a constant gain system.

Next, consider passivity of LTI systems in descriptor form. Such LTI systems are expressed as

$$
E \dot{z}=A z+B u, \quad y=C z+D u
$$

where $z$ is the system state, $y$ is the system output, $u$ is the system input, and the matrices $(E, A, B, C, D)$ describe the dynamics of this system. For regular descriptor systems, the transfer 
function matrix of the LTI system is $G(s)=C(s E-A)^{-1} B+D$. Passivity of an LTI system can equivalently be characterized as $G(s)+G^{*}(s) \geq 0$ for all $\operatorname{Re}\{s\} \geq 0{ }^{15}$. Extending the KalmanYakubovitch lemma for passive LTI systems (or equivalently, positive real transfer functions) to descriptor form, it follows that if there exist matrices, $P=P^{T}>0, L$ and $W$, which satisfy

$$
\begin{aligned}
& A^{T} P E+E^{T} P A=-L^{T} L \\
& E^{T} P B-C^{T}=-L^{T} W \\
& D+D^{T}=W^{T} W
\end{aligned}
$$

then the transfer function $G(s)$ is positive real. Note that for $E=I$, the conditions in Eq. (2) reduce to the necessary and sufficient conditions of the positive realness lemma ${ }^{12,13}$.

Dynamics of a flexible space structure with rate output provides an example of passive LTI systems. The dynamics of flexible space structures are typically expressed in second order form as

$$
M \ddot{x}+D \dot{x}+K x=F u
$$

where $x$ denotes an $n \times 1$ vector of displacements, $u$ denotes an $m \times 1$ vector of control inputs, $M, D, K$ are mass, damping and stiffness matrices of the system, respectively, and $F$ is an $n \times m$ input influence matrix describing the actuator force distribution. The mass matrix, $M$, is a symmetric, positive definite matrix for physical systems, and the damping and stiffness matrices are symmetric, positive semidefinite matrices. Using compatible rate sensors, collocated with the actuators, the $m \times 1$ vector of rate output of the system is given by $y=F^{T} \dot{x}$. Compatibility of sensors and actuators implies that angular rate sensors are used with torquers, and linear velocity sensors are used with force or thrust actuators. These conditions ensure that the output influence matrix is the transpose of the input influence matrix. The dynamic equations for second order models can be rewritten as

$$
\left[\begin{array}{cc}
I & 0 \\
0 & M
\end{array}\right]\left\{\begin{array}{l}
\dot{x} \\
\ddot{x}
\end{array}\right\}=\left[\begin{array}{cc}
0 & I \\
-K & -D
\end{array}\right]\left\{\begin{array}{l}
x \\
\dot{x}
\end{array}\right\}+\left[\begin{array}{l}
0 \\
F
\end{array}\right] u, \quad y=\left[\begin{array}{ll}
0 & F^{T}
\end{array}\right]\left\{\begin{array}{l}
x \\
\dot{x}
\end{array}\right\}
$$

that is, the descriptor form

$$
E \dot{z}=A z+B u, \quad y=C z
$$


where $z=\left[\begin{array}{l}x \\ \dot{x}\end{array}\right], E=\left[\begin{array}{cc}I & 0 \\ 0 & M\end{array}\right], A=\left[\begin{array}{cc}0 & I \\ -K & -D\end{array}\right], B=\left[\begin{array}{l}0 \\ F\end{array}\right]$ and $C=B^{T}$. Note that, the conditions for positive realness in Eq. (2) above are satisfied with $P=\left[\begin{array}{cc}K & 0 \\ 0 & M^{-1}\end{array}\right]$, $L=\left[\begin{array}{ll}0 & \sqrt{2} L_{D}\end{array}\right]$ and $W=0$, where $L_{D}$ is a Cholesky factorization of $D$, that is, $D=L_{D}^{T} L_{D}$. Note that the mass matrix is nonsingular, and the stiffness matrix must be nonsingular for complete observability with rate feedback. Thus, the matrices above satisfy the conditions of the lemma for passivity of descriptor systems. Note that symmetry and positive definiteness properties of the matrices $M, K$ and $D$ follow from the physics of flexible structure dynamics and do not depend on specific values of the mass, stiffness and damping parameters. Thus, dynamics of flexible structures with rate output are passive irrespective of the parametric uncertainties associated with the mass, stiffness and damping matrices of the system. Furthermore, truncation of the system dynamics to a smaller set of modes for reduced-order design models does not affect these passivity properties.

The control law for constant gain output feedback is $u=-G y$, where $G$ is a constant $m \times m$ output feedback gain matrix. The closed-loop system dynamics with output feedback are given by $E \dot{z}=\left(A-B G B^{T}\right) z$. If the system is strictly passive, stability of the closed-loop system is guaranteed by the passivity theorem ${ }^{15}$ for any output feedback gain matrix whose symmetric part is positive semidefinite. The main problem addressed in this paper is how to select a constant output gain matrix, whose symmetric part is positive semidefinite, for assigning dominant poles of a system to enhance closed-loop system response while ensuring over all closed-loop system stability. The sequential procedure for assigning desired closed-loop eigenpairs described in this paper assumes that the open-loop LTI system is strictly passive. Flexible space structures with zero frequency rigid body modes, or modes with no damping, do not satisfy this condition. To deal with such systems, static dissipative position and rate feedback controllers may be designed first to ensure strict passivity of an intermediate closed-loop system ${ }^{12}$, and the sequential approach may be applied to this system for eigensystem assignment. Furthermore, subsequent discussions assume that a low order control design model for plant dynamics, as opposed to the full order finite element matrices, is used in the control design process. The reduction may 
be achieved through a number of model reduction techniques, such as modal truncation and modal cost analysis.

\section{Sequential Procedure}

The task of assigning dominant poles of strictly passive LTI systems with output feedback gain matrices whose symmetric part is positive semidefinite is accomplished in a sequential manner. In each step of the sequential procedure, one self-conjugate pair of closed-loop eigenvalues is assigned to desired values while making sure that the previously assigned closedloop eigenvalues are not disturbed. The approach is an extension of the ideas in Refs. 9 and 20 to descriptor systems with output feedback. Generalized ordered real Schur transformations of the system matrices, $E$ and $A$, are employed in each step of the sequential procedure, using the following result from Ref. 21. Given two real matrices $E$ and $A$, there exist orthogonal transformations $L$ and $U$ such that the matrices $\widetilde{E}=L^{T} E U$ and $\widetilde{A}=L^{T} A U$ are in ordered real Schur form. This transformation is known as the generalized real Schur transformation. These transformations are used to move previously assigned eigenvalue pairs to the top left block of the

pair $(\widetilde{E}, \widetilde{A})$, and the structure of the new gain matrix is prescribed such that it only affects the eigenvalues in the lower bottom portion of the system matrices. At each step, after computing the gain matrix that assigns a pair of desired closed-loop eigenvalues, the intermediate closedloop matrix is transformed to a generalized Schur form with all previously assigned eigenpairs in the top left block of the updated system matrix. The overall output feedback gain matrix is constructed by accumulating the gains from each step. Since the symmetric part of each of the component matrices is positive semidefinite, the overall gain matrix also satisfies this robust stability condition. This process can be continued until $m$ closed-loop eigenvalues have been assigned to the desired locations.

The sequential procedure is conceptually straightforward and easy to implement in software since updated variables in the sequential process overwrite previous variables. However, description of the procedure below appears to be somewhat complicated due to notational 
details in expressing the algorithm. For initialization of the procedure, denote the original state coordinates as $z_{1}=z$, the system matrices in the original state coordinates as $E_{1}=E, A_{1}=A$, $B_{1}=B, C_{1}=C$, and, initially, the output gain matrix is $G_{0}=0$. At the $k^{t h}$ step of the sequential procedure, $(k-1)$ self-conjugate pairs of the closed-loop eigenvalues have been placed in the previous steps. A gain matrix $G_{k}$ is computed in this step to place the $k^{t h}$ pair of the desired closed-loop eigenvalues while making sure that the $(k-1)$ self-conjugate pairs assigned in the previous steps remain unchanged.

First, orthogonal matrices $L_{k}$ and $U_{k}$ are selected such that $\widetilde{E}_{k}=L_{k}^{T} E_{k} U_{k}$ and $\widetilde{A}_{k}=$ $L_{k}^{T} A_{k} U_{k}$ are in ordered real Schur form, and the $(k-1)$ self-conjugate eigenvalue pairs assigned in the previous steps are in the top left block of $\left(\widetilde{E}_{k}, \widetilde{A}_{k}\right)$. Applying these transformations to the system equations leads to $\widetilde{B}_{k}=L_{k}^{T} B_{k}$ and $\widetilde{C}_{k}=C_{k} U_{k}$. If $\widetilde{G}_{k}$ is the gain matrix at the $k^{t h}$ step, the closed-loop equations would be of the form $\widetilde{E}_{k} \dot{\widetilde{z}}_{k}=\left(\widetilde{A}_{k}-\widetilde{B}_{k} \widetilde{G}_{k} \widetilde{C}_{k}\right) \widetilde{z}_{k}$, where $\widetilde{z}_{k}=U_{k}^{T} z_{k}$ is the transformed state. The goal is to prescribe the structure for the gain matrix $\widetilde{G}_{k}$ such that previously placed eigenvalues are not disturbed by this gain matrix.

The matrices $\widetilde{E}_{k}, \widetilde{A}_{k}$ in ordered real Schur form are partitioned as follows:

$$
\widetilde{E}_{k}=\left[\begin{array}{cc}
\widetilde{E}_{k 11} & \widetilde{E}_{k 12} \\
0 & \widetilde{E}_{k 22}
\end{array}\right], \quad \widetilde{A}_{k}=\left[\begin{array}{cc}
\widetilde{A}_{k 11} & \widetilde{A}_{k 12} \\
0 & \widetilde{A}_{k 22}
\end{array}\right]
$$

where $\widetilde{E}_{k 11}, \widetilde{A}_{k 11}$ are $2(k-1) \times 2(k-1)$ matrices, $\widetilde{E}_{k 12}, \widetilde{A}_{k 12}$ are $2(k-1) \times 2(n-k+1)$ matrices, and $\widetilde{E}_{k 22}, \widetilde{A}_{k 22}$ are $2(n-k+1) \times 2(n-k+1)$ matrices. Note that the orthogonal matrices $L_{k}$ and $U_{k}$ have been selected such that the previously placed closed-loop eigenvalues are the eigenvalues of $\left(\widetilde{E}_{k 11}, \widetilde{A}_{k 11}\right)$, and the remaining eigenvalues of the system are eigenvalues of $\left(\widetilde{E}_{k 22}, \widetilde{A}_{k 22}\right)$. Corresponding partions of $\widetilde{B}_{k}$ and $\widetilde{C}_{k}$ are

$$
\widetilde{B}_{k}=\left[\begin{array}{l}
\widetilde{B}_{k 1} \\
\widetilde{B}_{k 2}
\end{array}\right], \quad \widetilde{C}_{k}=\left[\begin{array}{ll}
\widetilde{C}_{k 1} & \widetilde{C}_{k 2}
\end{array}\right]
$$

where $\widetilde{B}_{k 1}$ is a $2(k-1) \times m$ matrix, $\widetilde{B}_{k 2}$ is a $2(n-k+1) \times m$ matrix, $\widetilde{C}_{k 1}$ is a $m \times 2(k-1)$ matrix, and $\widetilde{C}_{k 2}$ is a $m \times 2(n-k+1)$ matrix.

Let $\widetilde{N}_{k}$ denote a matrix whose columns form an orthogonal basis for the left null space of $\widetilde{C}_{k 1}$, that is, $\widetilde{N}_{k}$ is a matrix with orthogonal columns such that $\widetilde{N}_{k}^{T} \widetilde{C}_{k 1}=0$. Let $m_{k}$ denote the 
left nullity of $\widetilde{C}_{k 1}$, so that $\widetilde{N}_{k}$ is an $m \times m_{k}$ matrix. If the gain matrix $\widetilde{G}_{k}$ is constructed as $\widetilde{G}_{k}=\widetilde{N}_{k} \widehat{G}_{k} \widetilde{N}_{k}^{T}$, where $\widehat{G}_{k}$ may be an arbitrary $m_{k} \times m_{k}$ matrix, then output feedback with $\widetilde{G}_{k}$ as the gain matrix will not affect the $(k-1)$ eigenvalue pairs assigned in the previous steps. To see this, consider the expanded form of the following closed-loop matrix,

$$
\left[\widetilde{A}_{k}-\widetilde{B}_{k} \widetilde{G}_{k} \widetilde{C}_{k}\right]=\left[\begin{array}{cc}
\widetilde{A}_{k 11} & \widetilde{A}_{k 12}-\widetilde{B}_{k 1} \widetilde{N}_{k} \widehat{G}_{k} \widetilde{N}_{k}^{T} \widetilde{C}_{k 2} \\
0 & \widetilde{A}_{k 22}-\widetilde{B}_{k 2} \widetilde{N}_{k} \widehat{G}_{k} \widetilde{N}_{k}^{T} \widetilde{C}_{k 2}
\end{array}\right]
$$

The first column of submatrices is not affected by the feedback since $\widetilde{N}_{k}^{T} \widetilde{C}_{k 1}=0$ by design. Therefore, eigenvalues of $\left(\widetilde{E}_{k}, \widetilde{A}_{k}-\widetilde{B}_{k} \widetilde{G}_{k} \widetilde{C}_{k}\right)$ are the union of the eigenvalues of $\left(\widetilde{E}_{k 11}, \widetilde{A}_{k 11}\right)$ and the eigenvalues of $\left(\widetilde{E}_{k 22}, \widetilde{A}_{k 22}-\widetilde{B}_{k 2} \widetilde{N}_{k} \widehat{G}_{k} \widetilde{N}_{k}^{T} \widetilde{C}_{k 2}\right)$, that is, the previously placed eigenvalues of $\left(\widetilde{E}_{k 11}, \widetilde{A}_{k 11}\right)$ remain unaffected by the output feedback.

The matrix $\widehat{G}_{k}$ is selected to place the $k^{\text {th }}$ self-conjugate pair of closed-loop eigenvalues, $\left(\lambda_{k}, \bar{\lambda}_{k}\right)$. Denoting $\widehat{E}_{k}=\widetilde{E}_{k 22}, \widehat{A}_{k}=\widetilde{A}_{k 22}, \widehat{B}_{k}=\widetilde{B}_{k 2} \widetilde{N}_{k}$, and $\widehat{C}_{k}=\widetilde{N}_{k}^{T} \widetilde{C}_{k 2}$, the problem is reduced to selecting an $m_{k} \times m_{k}$ output feedback gain matrix, $\widehat{G}_{k}$ for the descriptor system represented by the system matrices $\widehat{E}_{k}, \widehat{A}_{k}, \widehat{B}_{k}$, and $\widehat{C}_{k}$, such that $\left(\lambda_{k}, \bar{\lambda}_{k}\right)$ is an eigenvalue pair of the closed-loop system $\left(\widehat{E}_{k}, \widehat{A}_{k}-\widehat{B}_{k} \widehat{G}_{k} \widehat{C}_{k}\right)$. It must be assumed at this point that the controllable and observable subspaces for the system $\left(\widehat{A}_{k}, \widehat{B}_{k}, \widehat{C}_{k}\right)$ are not null. Note that, by construction, if the symmetric part of $\widehat{G}_{k}$ is positive semidefinite, then the symmetric part of $\widetilde{G}_{k}$ will be positive semidefinite, and if $\widehat{G}_{k}$ is symmetric and positive semidefinite, then $\widetilde{G}_{k}$ is symmetric and positive semidefinite. Thus, if $\widehat{G}_{k}$ satisfies the criteria for robustness, so does $\widetilde{G}_{k}$. The procedure for selecting $\widehat{G}_{k}$ is described in the next section.

Finally, the gain matrix is updated as $G_{k}=G_{k-1}+\widetilde{G}_{k}$, and the system matrices are updated as $E_{k+1}=\widetilde{E}_{k}, A_{k+1}=\left(\widetilde{A}_{k}-\widetilde{B}_{k} \widetilde{G}_{k} \widetilde{C}_{k}\right), B_{k+1}=\widetilde{B}_{k}$, and $C_{k+1}=\widetilde{C}_{k}$, for the succeeding steps of the sequential procedure. Note that $m_{k}$, the dimension of the left null space of $\widetilde{C}_{k 1}$ decreases by 2 in each step of the sequential procedure, that is, $m_{k}=m_{k-1}-2$.

The sequential procedure above is repeated until all the desired dominant modes have been shifted, up to a maximum of $m / 2$ modes or complex-conjugate pairs, where $m$ is the number of inputs. After $m / 2$ iterations of the procedure described above, $m_{k}$, the dimension of the left null space of $\widetilde{C}_{k 1}$ will be zero, and there would be no freedom to assign additional eigenvalues. 


\section{Eigenpair Assignment}

This section describes the approach to select output feedback gains to assign one pair of complex conjugate eigenvalues, while ensuring that (1) the symmetric part of the gain matrix is positive semidefinite, or (2) the gain matrix is symmetric and positive semidefinite. For notational simplicity, the system matrices will be denoted as $E, A, B, C$ (corresponding to $\widehat{E}_{k}, \widehat{A}_{k}, \widehat{B}_{k}, \widehat{C}_{k}$ of the previous section), the output feedback gain matrix will be denoted as $G$ (instead of $\widehat{G}_{k}$ of previous section), the number of inputs is denoted by $m$ (in place of $m_{k}$ ) and the desired eigenvalue pair will be denoted $(\lambda, \bar{\lambda})$ rather than $\left(\lambda_{k}, \bar{\lambda}_{k}\right)$. Therefore, in the simpler notation, the problem is to select a matrix $G$, with a specified structure for robustness, such that $(\lambda, \bar{\lambda})$ is a generalized eigenpair of the closed-loop system matrix, $(E, A-B G C)$.

Let $\phi$ be the closed-loop eigenvector corresponding to the eigenvalue $\lambda$. The generalized eigenvalue problem becomes $(\lambda E-A+B G C) \phi=0$. This closed-loop expression can be rewritten as

$$
[\lambda E-A \quad \mid \quad B]\left[\begin{array}{c}
\phi \\
-\frac{\phi}{G C \phi}
\end{array}\right] \equiv \Gamma\left[\begin{array}{c}
\phi \\
-\frac{\bar{G}}{G C \phi}
\end{array}\right]=0
$$

It is obvious from Eq. (9), that the vector on the right hand side of the expression above must lie in the right null space of $\Gamma$. Let $N$ be a matrix whose columns form an orthogonal basis for the null space of $\Gamma$, that is, $\Gamma N=0$. Note that though $E, A$ and $B$ are real matrices, $\Gamma$ and $N$ are complex matrices since the eigenvalue $\lambda$ is complex scalar. However, to ensure that the gain matrix is real the closed-loop eigenvector corresponding to the complex-conjugate eigenvalue is chosen to be the complex-conjugate of $\phi$, that is, $\bar{\phi}$ is chosen to be the eigenvector corresponding to $\bar{\lambda}$. The overbar in the expressions in this section refer to complex-conjugation of the elements of the corresponding vector (or matrix) only, as opposed to the Hermitian operator, which involves transposition and complex-conjugation.

Since columns of $N$ span the null space of $\Gamma$, it follows that

$$
\left[\begin{array}{c}
\phi \\
-\overline{G C} \bar{\phi}
\end{array}\right]=N \alpha=\left[\begin{array}{c}
N_{1} \\
-\overline{N_{2}}-
\end{array}\right] \alpha
$$


where $\alpha$ is an arbitrary vector of complex elements, and the matrices $N_{1}, N_{2}$ are formed by partitioning $N$ compatibly with $\phi$ and $G C \phi$. Therefore, $\phi=N_{1} \alpha$ and $G C \phi=N_{2} \alpha$, which leads to

$$
G C N_{1} \alpha=N_{2} \alpha
$$

The eigenassignment problem is now reduced to selecting $\alpha$ such that there exists a gain matrix, $G$, satisfying Eq. (11) whose symmetric part is positive semidefinite. With $\bar{\phi}$ being the eigenvector corresponding to $\bar{\lambda}$, real solutions for the gain matrix $G$ can be obtained, and the equations can be written out to involve only real arithmetic operations as follows.

For the eigenvalue, $\bar{\lambda}$, with closed-loop eigenvector, $\bar{\phi}$, the matrix $\bar{\Gamma}=[\bar{\lambda} E-A \quad \mid \quad B]$, and $\bar{N}$ is a matrix whose orthogonal columns span the null space of $\bar{\Gamma}$. If the arbitrary coefficient vector is chosen to be $\bar{\alpha}$, the complex-conjugate of $\alpha$, then it follows that

$$
G C \bar{N}_{1} \bar{\alpha}=\bar{N}_{2} \bar{\alpha}
$$

Eq. (11) and Eq. (12) can equivalently be rewritten as

$$
G C\left[\operatorname{Re}\left(N_{1}\right) \quad-\operatorname{Im}\left(N_{1}\right)\right]\left[\begin{array}{l}
\operatorname{Re}(\alpha) \\
\operatorname{Im}(\alpha)
\end{array}\right]=\left[\begin{array}{ll}
\operatorname{Re}\left(N_{2}\right) & -\operatorname{Im}\left(N_{2}\right)
\end{array}\right]\left[\begin{array}{c}
\operatorname{Re}(\alpha) \\
\operatorname{Im}(\alpha)
\end{array}\right]
$$

and

$$
G C\left[\operatorname{Im}\left(N_{1}\right) \quad \operatorname{Re}\left(N_{1}\right)\right]\left[\begin{array}{l}
\operatorname{Re}(\alpha) \\
\operatorname{Im}(\alpha)
\end{array}\right]=\left[\begin{array}{ll}
\operatorname{Im}\left(N_{2}\right) & \operatorname{Re}\left(N_{2}\right)
\end{array}\right]\left[\begin{array}{l}
\operatorname{Re}(\alpha) \\
\operatorname{Im}(\alpha)
\end{array}\right]
$$

where $\operatorname{Re}(\bullet)$ denotes real part of the argument, and $\operatorname{Im}(\bullet)$ denotes imaginary part of the argument. In compact form these equations are written out as

$$
\begin{aligned}
& G W_{1} p=V_{1} p \\
& G W_{2} p=V_{2} p
\end{aligned}
$$

where $p=[\operatorname{Re}(\alpha) ; \operatorname{Im}(\alpha)], W_{1}=C\left[\operatorname{Re}\left(N_{1}\right)-\operatorname{Im}\left(N_{1}\right)\right], V_{1}=\left[\operatorname{Re}\left(N_{2}\right)-\operatorname{Im}\left(N_{2}\right)\right]$, $W_{2}=C\left[\operatorname{Im}\left(N_{1}\right) \quad \operatorname{Re}\left(N_{1}\right)\right]$, and $V_{2}=\left[\operatorname{Im}\left(N_{2}\right) \quad \operatorname{Re}\left(N_{2}\right)\right]$. Note that Eq. (15) is a system of quadratic equations in the unknown variables, namely, the elements of the gain matrix, $G$, and the coefficient vector, $p$. Further, the elements of $G$ are constrained such that $\operatorname{sym}(G) \geq 0$. Solution of Eq. (15) provides the desired pole placement with dissipative gain matrices. The 
following proposition gives the necessary and sufficient conditions to be satisfied by a coefficient vector $p$ for the existence of a solution matrix $G$ of desired structure ${ }^{22}$.

Proposition 1. There exists a matrix $G$, whose symmetric part is positive semidefinite, that satisfies Eq. (15), if and only if, there exists a vector $p$ which satisfies

$$
\begin{gathered}
p^{T} V_{1}^{T} W_{1} p \geq 0 \\
p^{T} V_{2}^{T} W_{2} p \geq 0 \\
\left(p^{T} V_{1}^{T} W_{1} p\right)\left(p^{T} V_{2}^{T} W_{2} p\right)-\frac{1}{4}\left(p^{T} V_{1}^{T} W_{2} p+p^{T} V_{2}^{T} W_{1} p\right)^{2} \geq 0
\end{gathered}
$$

Furthermore, there exists a symmetric, positive semidefinite matrix $G$ which solves Eq. (15) if and only if there exists a vector $p$ which satisfies Eq. (16) and $p^{T} V_{1}^{T} W_{2} p=p^{T} V_{2}^{T} W_{1} p$.

Proof: Denote $y_{1}=V_{1} p, y_{2}=V_{2} p, x_{1}=W_{1} p$ and $x_{2}=W_{2} p$. Then, Eq. (15) leads to $G\left[\begin{array}{ll}x_{1} & x_{2}\end{array}\right]=\left[\begin{array}{ll}y_{1} & y_{2}\end{array}\right]$, and the conditions in Eq. (16) become $y_{1}^{T} x_{1} \geq 0, y_{2}^{T} x_{2} \geq 0$ and $\left(y_{1}^{T} x_{1}\right)\left(y_{2}^{T} x_{2}\right)-\frac{1}{4}\left(y_{1}^{T} x_{2}+y_{2}^{T} x_{1}\right)^{2} \geq 0$. Therefore,

$$
\begin{aligned}
{\left[\begin{array}{ll}
x_{1} & x_{2}
\end{array}\right]^{T} G\left[\begin{array}{ll}
x_{1} & x_{2}
\end{array}\right] } & =\left[\begin{array}{ll}
x_{1} & x_{2}
\end{array}\right]^{T}\left[\begin{array}{ll}
y_{1} & y_{2}
\end{array}\right] \\
& =\left[\begin{array}{ll}
x_{1}^{T} y_{1} & x_{1}^{T} y_{2} \\
x_{2}^{T} y_{1} & x_{2}^{T} y_{2}
\end{array}\right]
\end{aligned}
$$

Adding this relation and its transpose yields

$$
\left[\begin{array}{ll}
x_{1} & x_{2}
\end{array}\right]^{T}[\operatorname{sym}(G)]\left[\begin{array}{ll}
x_{1} & x_{2}
\end{array}\right]=\left[\begin{array}{cc}
y_{1}^{T} x_{1} & 0.5\left(y_{1}^{T} x_{2}+y_{2}^{T} x_{1}\right) \\
0.5\left(y_{1}^{T} x_{2}+y_{2}^{T} x_{1}\right) & y_{2}^{T} x_{2}
\end{array}\right]
$$

Now if $\operatorname{sym}(G) \geq 0$, then the equation above implies,

$$
\left[\begin{array}{cc}
y_{1}^{T} x_{1} & 0.5\left(y_{1}^{T} x_{2}+y_{2}^{T} x_{1}\right) \\
0.5\left(y_{1}^{T} x_{2}+y_{2}^{T} x_{1}\right) & y_{2}^{T} x_{2}
\end{array}\right] \geq 0
$$

Noting that determinants of principal minors of a positive semidefinite matrix must be nonnegative leads to conditions in Eq. (16). Thus, if there exists a matrix $G$, which satisfies Eq. (15), and its symmetric part is positive semidefinite, then the vector $p$ must satisfy conditions in Eq. (16).

Conversely, it has to be shown that if a vector $p$ satisfies the conditions in Eq. (16), then there exists a matrix $G$ which satisfies Eq. (15), whose symmetric part is positive semidefinite. 
This is proved by constructing such a matrix. Let $X=\left[\begin{array}{ll}x_{1} & x_{2}\end{array}\right]$ and $Y=\left[\begin{array}{ll}y_{1} & y_{2}\end{array}\right]$, then Eq. (15) is rewritten as $G X=Y$. Let $Q$ be an orthogonal matrix, such that

$$
Q^{T} Y=\left[\begin{array}{c}
\tilde{Y}_{1} \\
0
\end{array}\right]
$$

where $\tilde{Y}_{1}$ is a nonsingular $2 \times 2$ matrix. The matrix $Q$ can be obtained by $\mathrm{QR}$ factorization of $Y$. Now, define $\widetilde{X}_{1}, \widetilde{X}_{2}$ as follows

$$
\left[\begin{array}{c}
\widetilde{X}_{1} \\
\widetilde{X}_{2}
\end{array}\right]=Q^{T} X
$$

where $\widetilde{X}_{1}$ is a $2 \times 2$ matrix, and $\widetilde{X}_{2}$ is a $(m-2) \times 2$ matrix. Now $\widetilde{X}_{1}$ is nonsingular if $x_{1}$ and $x_{2}$ are linearly independent (otherwise, Eq. 15 is solved trivially). Defining $\widetilde{G}_{11}=\widetilde{Y}_{1} \widetilde{X}_{1}^{-1}$, it can be seen that

$$
\left[\begin{array}{cc}
\widetilde{G}_{11} & 0 \\
0 & 0
\end{array}\right]\left[\begin{array}{c}
\widetilde{X}_{1} \\
\widetilde{X}_{2}
\end{array}\right]=\left[\begin{array}{c}
\widetilde{Y}_{1} \\
0
\end{array}\right]
$$

Therefore, it follows that the matrix $G$ defined as $G=Q\left[\begin{array}{cc}\widetilde{G}_{11} & 0 \\ 0 & 0\end{array}\right] Q^{T}$ satisfies $G X=Y$.

Next it is shown that $\operatorname{sym}(G) \geq 0$. From Eq. (19) it follows that conditions in Eq. (16) imply $\operatorname{sym}\left(Y^{T} X\right) \geq 0$. Next, since $Y^{T} X=\left(Q^{T} Y\right)^{T}\left(Q^{T} X\right)=\tilde{Y}_{1}^{T} \widetilde{X}_{1}, \operatorname{sym}\left(Y^{T} X\right) \geq 0$ leads to $\operatorname{sym}\left(\tilde{Y}_{1}^{T} \widetilde{X}_{1}\right) \geq 0$. Noting that $\operatorname{sym}\left(\widetilde{Y}_{1}^{T} \widetilde{X}_{1}\right)=\widetilde{X}_{1}^{T}\left[\operatorname{sym}\left(\widetilde{G}_{11}\right)\right] \widetilde{X}_{1} \geq 0, \operatorname{sym}\left(\widetilde{G}_{11}\right) \geq 0$ follows from the nonsingularity of $\widetilde{X}_{1}$. Finally, by construction, $\operatorname{sym}(G) \geq 0$ if $\operatorname{sym}\left(\widetilde{G}_{11}\right) \geq 0$.

Furthermore, if $G$ is symmetric, that is, $G=\operatorname{sym}(G)$, then $y_{1}^{T} x_{2}=x_{1}^{T} G^{T} x_{2}=x_{2}^{T} G^{T} x_{1}=$ $y_{2}^{T} x_{1}$, so that the additional condition in the proposition is satisfied. On the other hand, the additional constraint ensures that $Y^{T} X=\tilde{Y}_{1}^{T} \widetilde{X}_{1}$ is symmetric. Since $\tilde{Y}_{1}=\widetilde{G}_{11} \tilde{X}_{1}$, $\tilde{Y}_{1}^{T} \widetilde{X}_{1}=\widetilde{X}_{1}^{T} \widetilde{Y}_{1}$, and $\widetilde{X}_{1}$ is nonsingluar, it follows that $\widetilde{G}_{11}$ is symmetric. Finally, again by construction, $G$ is symmetric when $\widetilde{G}_{11}$ is symmetric.

With this proposition, the problem of eigenpair assignment with dissipative output feedback is reduced to selection of a coefficient vector $p$ which satisfies the conditions in Eq. (16). A minimax approach to determining a coefficient vector $p$ which satisfies the conditions in Eq. (16) is described next. 
Note that if there exists a coefficient vector $p$ which satisfies

$$
\begin{aligned}
& p^{T} V_{1}^{T} W_{1} p-\left|\frac{1}{2}\left(p^{T} V_{1}^{T} W_{2} p+p^{T} V_{2}^{T} W_{1} p\right)\right| \geq 0 \\
& p^{T} V_{2}^{T} W_{2} p-\left|\frac{1}{2}\left(p^{T} V_{1}^{T} W_{2} p+p^{T} V_{2}^{T} W_{1} p\right)\right| \geq 0
\end{aligned}
$$

then it also satisfies the conditions in Eq. (16). The first two inequalities of Eq. (16) are obviously satisfied when the inequalities in Eq. (23) are satisfied, and multiplying the two inequalities in Eq. (23) shows that the third condition of Eq. (16) is also satisfied. Further, the inequalities in Eq. (23) can equivalently be written as four inequalities, which are quadratics in the coefficient vector, $p$, as follows

$$
\begin{aligned}
& f_{1}(p)=p^{T}\left\{V_{1}^{T} W_{1}+\frac{1}{2}\left(V_{1}^{T} W_{2}+V_{2}^{T} W_{1}\right)\right\} p \geq 0 \\
& f_{2}(p)=p^{T}\left\{V_{1}^{T} W_{1}-\frac{1}{2}\left(V_{1}^{T} W_{2}+V_{2}^{T} W_{1}\right)\right\} p \geq 0 \\
& f_{3}(p)=p^{T}\left\{V_{2}^{T} W_{2}+\frac{1}{2}\left(V_{1}^{T} W_{2}+V_{2}^{T} W_{1}\right)\right\} p \geq 0 \\
& f_{4}(p)=p^{T}\left\{V_{2}^{T} W_{2}-\frac{1}{2}\left(V_{1}^{T} W_{2}+V_{2}^{T} W_{1}\right)\right\} p \geq 0
\end{aligned}
$$

A feasible coefficient vector, $p$, can now be obtained maximizing the minimum of the four quadratics, $f_{i}(p), i=1, \ldots, 4$. This problem becomes a standard minimax problem by reversing the sign of the quadratics, that is, minimizing the maximum of $-f_{i}(p), i=1, \ldots, 4$. This problem is transformed to a constrained minimization problem by introducing a scalar variable, $\eta$, as follows ${ }^{22}$,

$$
\min _{p, \eta} \eta \quad \text { such that } f_{i}(p)+\eta \geq 0, i=1, \ldots, 4
$$

Standard nonlinear programming techniques may be used for this constrained minimization. Analytic gradients of $f_{i}(p)$ are readily available, since the gradient of any quadratic, $f(p)=$ $p^{T} Q p$, is given by $\frac{\partial}{\partial p} f(p)=\left(Q+Q^{T}\right) p$. The optimization of Eq. (25) is very well-behaved because the functions, $f_{i}(p), i=1, \ldots, 4$, are quadratic in $p$, and analytic gradients are linear in p. A nonpositive value of $\eta$ in Eq. (25) provides a feasible coefficient vector, $p$, that satisfies the conditions in Eq. (16) for existence of a gain matrix whose symmetric part is positive 
semidefinite. The convergence of the nonlinear optimization in Eq. (25) is not an issue, since the search can be terminated once a desirable target value of $\eta$ (which must be negative) has been attained. Furthermore, if a symmetric, positive semidefinite gain matrix is desired, the additional equality constraint required for symmetry (in Proposition 1) can also be included in the optimization of Eq. (25). Since this equality again involves a quadratic in $p$, its analytic gradients are linear and readily available.

Experience in application of the minimax approach presented above has shown that this technique is very effective in obtaining a feasible coefficient vector, $p$, which satisfies the conditions in Eq. (16). However, a number of other approaches have also been explored for this problem ${ }^{16,17}$. Another useful approach is to characterize a subspace for the vector $p$, which satisfies the first two conditions in Eq. (16). This can be accomplished by examining the eigenvectors corresponding the positive eigenvalues of the symmetric parts of the matrices $V_{1}^{T} W_{1}$ and $V_{2}^{T} W_{2}$. Then the function in the third inequality of Eq. (16) is maximized with $p$ being restricted to this subspace, until a positive value for that function is reached. This approach has also proved successful for a number of problems, though the minimax approach presented earlier has been successful in obtaining a feasible coefficient vector, $p$, for a larger class of problems.

Once such a coefficient vector has been determined, the procedure given in the proof of Proposition 1 can be followed to determine the gain matrix $G$, with positive semidefinite symmetric part, that assigns the desired closed-loop complex-conjugate eigenvalues.

\section{Position and Rate Feedback}

Pole placement for second order models, such as those for dynamics of flexible space structures, with both position and rate feedback is considered in this section. Position feedback gives additional freedom to place closed-loop eigenvalues, which may not be feasible with rate feedback alone. The technique for eigenpair placement with rate and position feedback parallels 
that for rate feedback alone, the difference being that the development is more involved since expressions for both rate and position feedback gain matrices are considered simultaneously.

Consider a second-order model, as shown in Eq. (3), again. Position sensors are now assumed to be collocated with the rate sensors and actuators to provide the position output. Thus, an $m \times 1$ vector of position output, $y_{p}$, is given by $y_{p}=C_{p} z$, where $C_{p}=\left[\begin{array}{ll}F^{T} & 0\end{array}\right]$. As before, the rate output is $y_{r}=C_{r} z$, where $C_{r}=\left[\begin{array}{ll}0 & F^{T}\end{array}\right]$.

The eigensystem assignment problem is to determine an $m \times m$ symmetric, positive semidefinite position gain matrix, $G_{p}$, and an $m \times m$ rate gain matrix, $G_{r}$, whose symmetric part is positive semidefinite, such that the control law $u=-G_{p} y_{p}-G_{r} y_{r}$ assigns dominant poles of the system to desired locations. In other words, to determine gain matrices $G_{p}$ and $G_{r}$ such that certain eigenvalues of $\left(E, A-B G_{p} C_{p}-B G_{r} C_{r}\right)$ are at desired locations. Again, it must be ensured that other closed-loop eigenvalues do not become unstable during output feedback eigensystem assignment with position and rate feedback. Using Lyapunov function arguments, it can be shown that if $G_{p}$ is a symmetric, positive semidefinite matrix, and the symmetric part of $G_{r}$ is positive semidefinite, then the closed-loop system is stable.

A sequential methodology is used for eigensystem assignment with position and rate feedback, extending the approach for rate feedback in previous sections. At each step of the process, one self-conjugate eigenpair is assigned, while employing a structure for the gain matrices such that previously placed eigenvalues are not disturbed. For initializing the sequential procedure, set $E_{1}=E, A_{1}=A, B_{p, 1}=B, B_{r, 1}=B, C_{p, 1}=C_{p}, C_{r, 1}=C_{r}$, the original coordinates as $z_{1}=z$, and, the output gain matrices as $G_{p, 0}=G_{r, 0}=0$. At the $k^{t h}$ step of the sequential procedure, orthogonal matrices $L_{k}$ and $U_{k}$ are selected such that $\widetilde{E}_{k}=L_{k}^{T} E_{k} U_{k}$ and $\widetilde{A}_{k}=L_{k}^{T} A_{k} U_{k}$ are in ordered real Schur form, and the $(k-1)$ selfconjugate eigenvalue pairs assigned in the previous steps are in the top left block of $\left(\widetilde{E}_{k}, \widetilde{A}_{k}\right)$. Applying these transformations to the system matrices leads to $\widetilde{B}_{p, k}=L_{k}^{T} B_{p, k}, \widetilde{B}_{r, k}=L_{k}^{T} B_{r, k}$, $\widetilde{C}_{p, k}=C_{p, k} U_{k}$ and $\widetilde{C}_{r, k}=C_{r, k} U_{k}$. Partition $\widetilde{C}_{p, k}$ and $\widetilde{C}_{r, k}$ as follows: $\widetilde{C}_{p, k}=\left[\begin{array}{ll}\widetilde{C}_{p, k 1} & \widetilde{C}_{p, k 2}\end{array}\right]$ and $\widetilde{C}_{r, k}=\left[\begin{array}{ll}\widetilde{C}_{r, k 1} & \widetilde{C}_{r, k 2}\end{array}\right]$, where $\widetilde{C}_{p, k 1}, \widetilde{C}_{r, k 1}$ are $m \times 2(k-1)$ matrices. System matrices $\widetilde{E}_{k}, \widetilde{A}_{k}, \widetilde{B}_{p, k}$ and $\widetilde{B}_{r, k}$ are also partitioned conformally. Let the columns of $\widetilde{N}_{p, k}$ form an 
orthogonal basis for left null space of $\widetilde{C}_{p, k 1}$, and let the columns of $\widetilde{N}_{r, k}$ form an orthogonal basis for left null space of $\widetilde{C}_{r, k 1}$. Form the rate and position gain matrices for the $k^{\text {th }}$ step as follows: $\widetilde{G}_{p, k}=\widetilde{N}_{p, k} \widehat{G}_{p, k} \widetilde{N}_{p, k}^{T}$, where $\widehat{G}_{p, k}$ is an arbitrary matrix to be defined; and $\widetilde{G}_{r, k}=\widetilde{N}_{r, k} \widehat{G}_{r, k} \widetilde{N}_{r, k}^{T}$, where $\widehat{G}_{r, k}$ is an arbitrary matrix to be defined. Then, the matrices $\widetilde{G}_{p, k}$ and $\widetilde{G}_{r, k}$ do not affect the previously placed eigenvalues. To see this, consider the expanded form of the closed-loop system matrix, $\left(\widetilde{A}_{k}-\widetilde{B}_{p, k} \widetilde{G}_{p, k} \widetilde{C}_{p, k}-\widetilde{B}_{r, k} \widetilde{G}_{r, k} \widetilde{C}_{r, k}\right)$, shown below.

$$
\left[\begin{array}{cc}
\widetilde{A}_{k 11} & \widetilde{A}_{k 12}-\widetilde{B}_{p, k 1} \widetilde{N}_{p, k} \widehat{G}_{p, k} \widetilde{N}_{p, k}^{T} \widetilde{C}_{p, k 2}-\widetilde{B}_{r, k 1} \widetilde{N}_{r, k} \widehat{G}_{r, k} \widetilde{N}_{r, k}^{T} \widetilde{C}_{r, k 2} \\
0 & \widetilde{A}_{k 22}-\widetilde{B}_{p, k 2} \widetilde{N}_{p, k} \widehat{G}_{p, k} \widetilde{N}_{p, k}^{T} \widetilde{C}_{p, k 2}-\widetilde{B}_{r, k 2} \widetilde{N}_{r, k} \widehat{G}_{r, k} \widetilde{N}_{r, k}^{T} \widetilde{C}_{r, k 2}
\end{array}\right]
$$

The first column of submatrices remains unaffected by the feedback because $\tilde{N}_{p, k}^{T} \widetilde{C}_{p, k 1}=0$ and $\widetilde{N}_{r, k}^{T} \widetilde{C}_{r, k 1}=0$. Thus the eigenvalues of the closed-loop system with position and rate feedback of this form are the union of the eigenvalues of $\left(\widetilde{E}_{k 11}, \widetilde{A}_{k 11}\right)$, which are the previously placed eigenvalues, and the eigenvalues of $\left(\widetilde{E}_{k 22}, \widetilde{A}_{k 22}-\widetilde{B}_{p, k 2} \widetilde{N}_{p, k} \widehat{G}_{p, k} \widetilde{N}_{p, k}^{T} \widetilde{C}_{p, k 2}-\widetilde{B}_{r, k 2} \widetilde{N}_{r, k} \widehat{G}_{r, k} \widetilde{N}_{r, k}^{T} \widetilde{C}_{r, k 2}\right)$. Denoting $\widehat{E}_{k}=\widetilde{E}_{k 22}$, $\widehat{A}_{k}=\widetilde{A}_{k 22}, \widehat{B}_{p, k}=\widetilde{B}_{p, k 2} \widetilde{N}_{p, k}, \widehat{B}_{r, k}=\widetilde{B}_{r, k 2} \widetilde{N}_{r, k}, \widehat{C}_{p, k}=\widetilde{N}_{p, k}^{T} \widetilde{C}_{p, k 2}$, and $\widehat{C}_{r, k}=\widetilde{N}_{r, k}^{T} \widetilde{C}_{r, k 2}$, the problem is to select matrices $\widehat{G}_{p, k}$ and $\widehat{G}_{r, k}$ that satisfy the closed-loop stability constraints and that $\left(\widehat{E}_{k}, \widehat{A}_{k}-\widehat{B}_{p, k} \widehat{G}_{p, k} \widehat{C}_{p, k}-\widehat{B}_{r, k} \widehat{G}_{r, k} \widehat{C}_{r, k}\right)$ has one self-conjugate pair at the desired values. The gain matrices are updated as $G_{p, k}=G_{p, k-1}+\widetilde{G}_{p, k}$ and $G_{r, k}=G_{r, k-1}+\widetilde{G}_{r, k}$, and the system matrices are updated for the succeeding steps as: $E_{k+1}=\widetilde{E}_{k}, A_{k+1}=\left(\widetilde{A}_{k}-\widetilde{B}_{p, k} \widetilde{G}_{p, k} \widetilde{C}_{p, k}-\widetilde{B}_{r, k} \widetilde{G}_{r, k} \widetilde{C}_{r, k}\right), B_{p, k+1}=\widetilde{B}_{p, k}, B_{r, k+1}=\widetilde{B}_{r, k}$, $C_{p, k+1}=\widetilde{C}_{p, k}$ and $C_{r, k+1}=\widetilde{C}_{r, k}$. This procedure may be repeated up to $m / 2$ times, where $m$ is the number of inputs to the system.

For notational simplicity, consider synthesis of position and rate gain matrices $G_{p}, G_{r}$ for eigenplacement of the system given by the matrices $E, A, B_{p}, B_{r}, C_{p}, C_{r}$. In other words, the problem has been reduced to selecting a symmetric, positive semidefinite position gain matrix, $G_{p}$, and a rate gain matrix, $G_{r}$, whose symmetric part is positive semidefinite, such that $(\lambda, \bar{\lambda})$ is an eigenpair of the generalized eigenvalue problem $\left(E, A-B_{p} G_{p} C_{p}-B_{r} G_{r} C_{r}\right)$. Let $\phi$ be 
the closed-loop eigenvector such that $\left(\lambda E-A+B_{p} G_{p} C_{p}+B_{r} G_{r} C_{r}\right) \phi=0$, or equivalently,

$$
\left[\lambda E-A \quad\left|\quad B_{p} \quad\right| \quad B_{r}\right]\left[\begin{array}{c}
\phi \\
\overline{G_{p} C_{p} \phi} \\
\overline{G_{r} C_{r} \phi}
\end{array}\right] \equiv \Gamma\left[\begin{array}{c}
\phi \\
\overline{G_{p}} \overline{C_{p} \phi} \\
\overline{-}-\overline{G_{r}} \bar{r} \boldsymbol{\phi}
\end{array}\right]=0
$$

Let $N$ be a matrix whose columns form the right null space of $\Gamma$. Then, solution to Eq. (27) may be written as

$$
\left[\begin{array}{c}
\phi \\
\overline{G_{p}}-\overline{C_{p} \phi} \\
\overline{G_{r} C_{r} \phi}
\end{array}\right]=\left[\begin{array}{c}
N_{1} \\
-\overline{N_{p}}- \\
-\overline{N_{r}}-
\end{array}\right] \alpha
$$

where $N_{1}, N_{p}$ and $N_{r}$ are conformal partitions of the matrix $N$, and $\alpha$ is an arbitrary coefficient vector. Eq. (28) leads to

$$
\begin{aligned}
& G_{p} C_{p} N_{1} \alpha=N_{p} \alpha \\
& G_{r} C_{r} N_{1} \alpha=N_{r} \alpha
\end{aligned}
$$

Similar treatment for the complex-conjugate eigenvalue, $\bar{\lambda}$, and a corresponding eigenvector, $\bar{\phi}$, leads to

$$
\begin{aligned}
& G_{p} C_{p} \bar{N}_{1} \bar{\alpha}=\bar{N}_{p} \bar{\alpha} \\
& G_{r} C_{r} \bar{N}_{1} \bar{\alpha}=\bar{N}_{r} \bar{\alpha}
\end{aligned}
$$

Combining these equations to restrict all operations to real arithmetic results in

$$
\begin{array}{ll}
G_{r} W_{r 1} p=V_{r 1} p & G_{p} W_{p 1} p=V_{p 1} p \\
G_{r} W_{r 2} p=V_{r 2} p & G_{p} W_{p 2} p=V_{p 2} p
\end{array}
$$

where $p=[\operatorname{Re}(\alpha) ; \quad \operatorname{Im}(\alpha)] ; W_{r 1}=C_{r}\left[\operatorname{Re}\left(N_{1}\right) \quad-\operatorname{Im}\left(N_{1}\right)\right] ; V_{r 1}=\left[\begin{array}{lll}\operatorname{Re}\left(N_{r}\right) & -\operatorname{Im}\left(N_{r}\right)\end{array}\right]$; $W_{r 2}=C_{r}\left[\operatorname{Im}\left(N_{1}\right) \quad \operatorname{Re}\left(N_{1}\right)\right] ; V_{r 2}=\left[\operatorname{Im}\left(N_{r}\right) \quad \operatorname{Re}\left(N_{r}\right)\right] ; W_{p 1}=C_{p}\left[\operatorname{Re}\left(N_{1}\right)-\operatorname{Im}\left(N_{1}\right)\right] ;$ $V_{p 1}=\left[\begin{array}{lll}\operatorname{Re}\left(N_{p}\right) & -\operatorname{Im}\left(N_{p}\right)\end{array}\right] ; W_{p 2}=C_{p}\left[\operatorname{Im}\left(N_{1}\right) \quad \operatorname{Re}\left(N_{1}\right)\right] ;$ and $V_{p 2}=\left[\operatorname{Im}\left(N_{p}\right) \quad \operatorname{Re}\left(N_{p}\right)\right]$. Thus, the problem of eigensystem placement with rate and position feedback is reduced to selecting a coefficient vector $p$, a symmetric, positive semidefinite matrix, $G_{p}$, and a matrix, $G_{r}$, whose symmetric part is positive semidefinite which satisfy Eq. (31). The conditions for existence of a solution to this system of quadratic equations is given in the following proposition. 
Proposition 2. There exists a symmetric, positive semidefinite matrix, $G_{p}$, and a matrix, $G_{r}$, whose symmetric part is positive semidefinite, which satisfy Eq. (31), if and only if, there exists a vector $p$ that satisfies

$$
\begin{array}{cc}
p^{T} V_{p 1}^{T} W_{p 1} p \geq 0 & p^{T} V_{r 1}^{T} W_{r 1} p \geq 0 \\
p^{T} V_{p 2}^{T} W_{p 2} p \geq 0 & p^{T} V_{r 2}^{T} W_{r 2} p \geq 0 \\
\left(p^{T} V_{p 1}^{T} W_{p 1} p\right)\left(p^{T} V_{p 2}^{T} W_{p 2} p\right)-\frac{1}{4}\left(p^{T} V_{p 1}^{T} W_{p 2} p+p^{T} V_{p 2}^{T} W_{p 1} p\right)^{2} \geq 0 \\
\left(p^{T} V_{r 1}^{T} W_{r 1} p\right)\left(p^{T} V_{r 2}^{T} W_{r 2} p\right)-\frac{1}{4}\left(p^{T} V_{r 1}^{T} W_{r 2} p+p^{T} V_{r 2}^{T} W_{r 1} p\right)^{2} \geq 0 \\
p^{T} V_{p 1}^{T} W_{p 2} p=p^{T} V_{p 2}^{T} W_{p 1} p
\end{array}
$$

The proof of this proposition follows that of Proposition 1 for rate feedback.

Again, a minimax approach can be employed to determine a feasible coefficient vector, $p$, which would satisfy the conditions of Eq. (32). It can be easily verified that a vector $p$ satisfies these conditions if it satisfies the following eight quadratic inequalities,

$$
\begin{aligned}
& f_{1}(p)=p^{T}\left\{V_{p 1}^{T} W_{p 1}+\frac{1}{2}\left(V_{p 1}^{T} W_{p 2}+V_{p 2}^{T} W_{p 1}\right)\right\} p \geq 0 \\
& f_{2}(p)=p^{T}\left\{V_{p 1}^{T} W_{p 1}-\frac{1}{2}\left(V_{p 1}^{T} W_{p 2}+V_{p 2}^{T} W_{p 1}\right)\right\} p \geq 0 \\
& f_{3}(p)=p^{T}\left\{V_{p 2}^{T} W_{p 2}+\frac{1}{2}\left(V_{p 1}^{T} W_{p 2}+V_{p 2}^{T} W_{p 1}\right)\right\} p \geq 0 \\
& f_{4}(p)=p^{T}\left\{V_{p 2}^{T} W_{p 2}-\frac{1}{2}\left(V_{p 1}^{T} W_{p 2}+V_{p 2}^{T} W_{p 1}\right)\right\} p \geq 0 \\
& f_{5}(p)=p^{T}\left\{V_{r 1}^{T} W_{r 1}+\frac{1}{2}\left(V_{r 1}^{T} W_{r 2}+V_{r 2}^{T} W_{r 1}\right)\right\} p \geq 0 \\
& f_{6}(p)=p^{T}\left\{V_{r 1}^{T} W_{r 1}-\frac{1}{2}\left(V_{r 1}^{T} W_{r 2}+V_{r 2}^{T} W_{r 1}\right)\right\} p \geq 0 \\
& f_{7}(p)=p^{T}\left\{V_{r 2}^{T} W_{r 2}+\frac{1}{2}\left(V_{r 1}^{T} W_{r 2}+V_{r 2}^{T} W_{r 1}\right)\right\} p \geq 0 \\
& f_{8}(p)=p^{T}\left\{V_{r 2}^{T} W_{r 2}-\frac{1}{2}\left(V_{r 1}^{T} W_{r 2}+V_{r 2}^{T} W_{r 1}\right)\right\} p \geq 0
\end{aligned}
$$

and the equality constraint ensuring symmetry of the position gain matrix. A vector $p$ satisfying these conditions can be determined by maximizing the minimum of $f_{i}(p), i=1, \ldots, 8$. This is accomplished by performing a constrained minimization, similar to that of Eq. (25), with the additional equality constraint for symmetry of the position gain matrix. This minimax 
approach for determining feasible coefficient vectors $p$ satisfying the conditions of Eq. (32) has been very successful in practical application, though a number of other techniques have been attempted ${ }^{16,17}$. Once a coefficient vector satisfying the constraints in Eq. (32) has been determined, the procedure to construct the desired matrices also follows the same approach as for rate feedback.

\section{Numerical Example}

The approach for eigensystem assignment with dissipative gains has been applied for synthesis of controllers to enhance the damping of the phase 2 CSI Evolutionary Model (CEM), a testbed for control of flexible space structures at NASA Langley. Damping enhancement control for this structure represents a typical problem in the control of flexible space structures, since this structure possesses numerous, closely spaced, low-frequency modes, with uncertain modal parameters. Damping enhancement is required since inherent damping of the structure is very low. However, general output feedback pole placement techniques for increasing damping in the dominant low frequency modes have a tendency to destabilize the remaining modes of the system. When collocated rate sensors and actuators are used, the dynamics of the structure are passive, and the technique described in this paper can be applied for damping enhancement of the dominant modes, while ensuring stability of the overall closed-loop system.

The phase 2 CEM structure consists of a 62-bay central truss (each bay is 10 inches long), along with two horizontal booms for suspension, a vertical laser, and a vertical reflector tower, as shown in Fig. 1. This structure has 10 modes with frequencies up to about $5 \mathrm{~Hz}$., and 95 modes with frequencies under $60 \mathrm{~Hz}$. The first six modes are rigid body modes, due to suspension of the structure from the laboratory ceiling, that have frequencies up to about $0.3 \mathrm{~Hz}$. Eight control stations housing collocated and compatible sensors and actuators are located at the bays shown in Fig. 1. Air thrusters providing linear forces are available at these locations, along the directions shown in Fig. 1, providing an $8 \times 1$ control input vector, $u$. Linear velocities are 
assumed to be available at these locations, along the same directions, giving a $8 \times 1$ measurement vector, $y$. The problem is to determine an $8 \times 8$ rate gain matrix, $G$, whose symmetric part is positive semidefinite, such that the control law $u=-G y$ places a subset of the closed-loop eigenvalues at desired locations without destabilizing the remaining modes of the structure.

A control design model of the first 10 modes of the structure is used for this numerical example. Low inherent damping ratio of 0.1 percent has been assumed for the open-loop system. The open-loop eigenvalues along with damping and frequencies are shown in Table 1. Modal displacements at the eight sensor/ actuator locations are shown in Table 2. Since $m=8$ control inputs are available, it is possible to assign desired amount of damping to up to 4 modes.

The first objective was to increase damping ratio in the first mode to $10 \%$ that is, the first desired pair of closed-loop eigenvalues was $\lambda_{1,2}=-0.0818 \pm 0.8139 j$. Proceeding as described above, the system of quadratic equations in Eq. (15) was constructed for this eigenassignment. Then, a coefficient vector, $p$, which satisfies the conditions in Eq. (16) was determined as

$$
\begin{array}{r}
p=[1.0,1.0,1.0,-1.0,-1.0,1.0,-1.0,1.0,1.0 \\
\quad-0.2757,0.8621,-1.0,1.0,-1.0,1.0,-1.0]
\end{array}
$$

using the minimax approach. Upper bounds on components of the coefficient vector, $p$, were set to 1.0 , and lower bounds were set to -1.0 , for the constrained optimization problem in determining this coefficient vector. Proceeding as described in the proof of Proposition 1, a gain matrix $G_{1}$ for this eigenplacement was computed. Nonzero eigenvalues of the symmetric part of $G_{1}$ are 0.4129 and 0.8035 , which demonstrates that the symmetric part of $G_{1}$ is positive semidefinite. Closed-loop eigenvalues with this gain matrix are shown in Table 3. It should be observed that the gain matrix, $G_{1}$, successfully increased damping ratio of the first mode to 10 $\%$ without destabilizing other modes. In fact, damping is increased in most other modes due to the dissipative nature of the controller.

For the second step, damping ratio in the second mode of the system was to be increased to $10 \%$ resulting in the second pair of desired complex-conjugate eigenvalues to 
be $\lambda_{3,4}=-0.0830 \pm 0.8259 j$. A coefficient vector that satisfies the necessary and sufficient conditions for eigenplacement in Eq. (16) was determined as

$$
\begin{gathered}
p=[1.0,1.0,1.0,0.7088,1.0,-1.0,-1.0,-1.0,-1.0, \\
-0.5250,-1.0,1.0]
\end{gathered}
$$

Note that the dimension of the coefficient vector, $p$, for the second step has decreased by 4 as compared to the first step, since the dimension of the left null space of $\widetilde{C}_{12}$ for the second step has decreased by 2 , that is, $m_{2}=6$. The cumulative gain matrix $G_{2}$ for placing two eigenpairs has nonzero eigenvalues of the symmetric part at $0.2090,0.6899,0.9358$ and 1.8008 , which demonstrates the stability constraint on the structure of gain matrices is satisfied. The closedloop eigenvalues with the gain matrix, $G_{2}$, are presented in Table 4 , showing that both pair of complex conjugate eigenvalues are successfully placed without destabilizing any other modes.

In the third step, damping ratio of the third mode was increased to $10 \%$ which leads to the third pair of complex-conjugate eigenvalues as $\lambda_{5,6}=-0.0857 \pm 0.8522 j$. Repeating the operations for the third step, a coefficient vector which satisfies the conditions in Eq. (16) was

$$
p=[-1.0,1.0,-1.0,-0.9664,-1.0,1.0,-1.0,0.5762]
$$

which led to the cumulative gain matrix of $G_{3}$ for the third step with nonzero eigenvalues of the symmetric part at $0.0961,0.2843,0.7060,0.9694,1.9922$ and 4.2001 . Closed-loop eigenvalues with gain $G_{3}$ are shown in Table 5. Again, damping ratio in the first three modes has been increased exactly to $10 \%$ as desired without destabilizing any other modes. In fact, damping in most other modes has increased, with mode 6 being overdamped. Furthermore, modes $4,5,7$ and 8 are also very well damped.

With 8 control actuators, it is possible to increase damping in one other mode. Therefore, mode 9 was chosen for an increase in its damping ratio to $10 \%$ that is, the final pair of desired closed-loop eigenvalues was chosen as $\lambda_{7,8}=-1.8692 \pm 18.5982 j$. A feasible coefficient vector for this case was determined to be $p=[-1.0,-1.0,-0.7275,0.0497]$. The final gain matrix, 
$G$, that places four desired closed-loop eigenpairs, without destabilizing other higher frequency modes, was computed to be

$$
G=G_{4}=\left[\begin{array}{cccccccc}
0.7471 & 0.2028 & -0.3887 & -0.8128 & -0.2086 & 0.1163 & -0.3025 & -0.9071 \\
0.3282 & 1.1480 & 0.2282 & -1.4228 & 0.9391 & 0.1604 & -1.1181 & -0.1716 \\
-0.5818 & -0.4845 & 1.1284 & 1.5011 & -0.1217 & -0.4460 & 0.0589 & 0.2609 \\
-0.4390 & -0.8793 & -0.3065 & 3.1249 & -1.3062 & 0.4245 & 1.1933 & 0.2856 \\
0.4710 & 1.2129 & -0.7990 & -1.4584 & 4.1617 & 0.4467 & -3.9649 & 0.1294 \\
0.4000 & 0.1577 & -0.5367 & -0.8686 & -0.1063 & 0.3813 & 0.1086 & -0.3153 \\
-0.7939 & -1.3759 & 1.1685 & 1.7430 & -4.1583 & -0.6551 & 4.5028 & 0.2142 \\
-0.8909 & 0.0902 & 0.3491 & 0.3117 & 0.3107 & -0.0493 & 0.4911 & 1.2872
\end{array}\right]
$$

Eigenvalues of the symmetric part of the overall gain matrix, $G$, are $0.0043,0.0969,0.1242,0.5703,1.0164,1.9239,3.0166$ and 9.7289 , that is, $\operatorname{sym}(G)>0$. Closed-loop eigenvalues with the overall gain matrix is shown in Table 6, showing that all four closed-loop eigenvalue pairs have been placed at their desired locations, without destabilizing any of the higher frequency modes. In fact, damping has tended to increase in most other modes. This example clearly demonstrates the effectiveness of the proposed dissipative controller design technique for robust eigensystem assignment.

Results of damping enhancement control with both position and rate feedback are presented next. First, damping ratio of mode 1 was increased to $10 \%$. Following a procedure outlined in the previous section, a symmetric, positive semidefinite position gain matrix, were determined to assign the closed-loop pair. Closed-loop eigenvalues with these rate and position gain matrices are shown in Table 7. Proceeding with the sequential procedure, the damping ratios of modes 2, 3 and 7 were increased to $10 \%$. The eigensystem assignment procedure resulted in the following cumulative symmetric, positive definite position gain matrix,

$$
G_{p}=\left[\begin{array}{cccccccc}
3.4841 & -0.1125 & -4.6653 & 0.2965 & -0.2298 & 3.1246 & 1.1816 & -1.9022 \\
-0.1125 & 1.9084 & -0.5832 & -1.6722 & 7.2152 & 0.1647 & -6.5656 & -0.1680 \\
-4.6653 & -0.5832 & 7.9072 & 0.3844 & 1.5834 & -4.2352 & -3.1930 & 2.7889 \\
0.2965 & -1.6722 & 0.3844 & 1.7415 & -6.4787 & 0.1705 & 6.4519 & -0.1206 \\
-0.2298 & 7.2152 & 1.5834 & -6.4787 & 46.6525 & -0.2519 & -45.6357 & 0.2129 \\
3.1246 & 0.1647 & -4.2352 & 0.1705 & -0.2519 & 3.3434 & 1.2556 & -1.8501 \\
1.1816 & -6.5656 & -3.1930 & 6.4519 & -45.6357 & 1.2556 & 46.9748 & -1.4609 \\
-1.9022 & -0.1680 & 2.7889 & -0.1206 & 0.2129 & -1.8501 & -1.4609 & 1.4274
\end{array}\right]
$$


and a rate gain matrix, whose symmetric part is positive definite,

$$
G_{r}=\left[\begin{array}{cccccccc}
0.6007 & -0.7380 & -0.6090 & 0.7122 & -0.4572 & 0.6321 & -1.0302 & 0.6469 \\
0.4598 & 0.4789 & 0.4392 & 0.0355 & 1.6212 & 0.5455 & 0.0844 & -1.3040 \\
-0.4611 & -0.3645 & 1.0371 & 0.5755 & -0.3726 & -0.1362 & 0.9946 & 0.3222 \\
-0.7028 & -0.7606 & -0.3794 & 0.8393 & -0.6959 & -1.4334 & 0.3812 & 1.6530 \\
0.9575 & -0.3045 & -0.4236 & -0.5543 & 3.5101 & 0.5159 & -3.4491 & -0.6720 \\
0.2021 & -0.7357 & -0.9152 & 1.4169 & -0.0963 & 0.6254 & -0.6292 & 0.3972 \\
0.3593 & -1.0655 & -0.1207 & 0.9327 & -2.6389 & 0.1686 & 3.3490 & -0.4591 \\
-1.2277 & 1.3110 & 0.0893 & -1.6916 & 0.2233 & -0.6664 & 0.5661 & 0.3660
\end{array}\right]
$$

Table 8 shows the closed-loop system eigenvalues using the rate and position gain matrices obtained. It is observed that the desired closed-loop eigenvalues have been placed while maintaining stability of the other modes. Comparing the frequencies of the closed-loop poles, it is noted that some of the system frequencies have been shifted significantly from their open-loop values. Position gain brings about this shift in frequencies of the open-loop system, as may be anticipated. On the contrary, frequencies of the closed-loop system with rate feedback alone, in Tables 3-6, remain close to the open-loop values. This demonstrates one of the benefits of using both position and rate feedback, namely, significant shift in open-loop frequencies is possible if desired. Moreover, inclusion of the position feedback provides additional freedom beyond eigenvalue assignment such that improved controller designs are possible. 


\section{Concluding Remarks}

This paper presents a novel approach for robust eigensystem assignment using constant gain output feedback controllers with dissipativity constraints, that is, output feedback eigensystem with gain matrices whose symmetric parts are positive semidefinite. Robust eigensystem assignment in this context implies that stability of the closed-loop system is guaranteed, whereas in general eigensystem assignment using output feedback may destabilize the closed-loop system. This approach can be used for robust output eigensystem assignment for any passive LTI system in descriptor form. Specifically, it has been applied to second-order models of flexible structures with rate feedback. A sequential procedure is used to place one pair of complex conjugate closedloop eigenvectors in each step of the procedure, while ensuring that the previously assigned eigenvalues are not disturbed using generalized Schur transformations. The problem of assigning one pair of complex-conjugate closed-loop eigenvalues with gain matrices of a specified structure is reduced to a constrained solution of a system of quadratic equations. Necessary and sufficient conditions for the existence of a solution to these equations are presented in terms of inequality constraints on a coefficient vector. A minimax approach is presented to determine coefficient vectors which satisfy these constraints. It is shown that the sequential procedure can be used to assign up to $m$ self-conjugate closed-loop eigenvalues, where $m$ is the number of control inputs. For second-order models of flexible space structures, the approach has been extended for robust eigensystem assignment with both position and rate feedback. A numerical example of damping enhancement for a large flexible structure has been presented to demonstrate the approach. This example clearly demonstrates that the proposed approach provides a practical technique to design controllers that enhance the performance of the open-loop system while ensuring that the overall system remains stable.

\section{References}

[1] Moore, B. C., "On the Flexibility Offered by State Feedback in Multivariable Systems 
Beyond Closed-Loop Eigenvalue Assignments," IEEE Trans. on Automatic Control, Vol. 21, No. 5, September-October 1976, pp. 689-692.

[2] Klien, G., and Moore, B. C., "Eigenvalue-Generalized Eigenvector Assignment with State Feedback," IEEE Trans. on Automatic Control, Vol. 22, No. 1, January-February 1977, pp. $140-141$.

[3] Kautsky, J., Nichols, N. K., and Van Dooren, P., "Robust Pole Assignment in Linear State Feedback," International Journal of Control, Vol. 41, No. 5, July 1985, pp. 1129-1155.

[4] Davison, E. J., and Wang, S. H., "On Pole Assignment in Linear Multivariable Systems Using Output Feedback," IEEE Trans. on Automatic Control, Vol. 20, No. 4, July-August 1975, pp. 516-518.

[5] Fletcher, L., and Magni, J. F., "Exact Pole Assignment by Output Feedback, Part 1," International Journal of Control, Vol. 45, 1987, pp. 1995-2007.

[6] Misra, P., and Patel, R. V., "Numerical Algorithms for Eigenvalue Assignment by Constant and Dynamic Output Feedback," IEEE Trans. on Automatic Control, Vol. 34, No. 6, June 1989, pp. 579-588.

[7] Kimura, H., "A Further Result on the Problem of Pole Assignment by Output Feedback," IEEE Trans. on Automatic Control, Vol. 22, No. 3, May-June 1977, pp. 458-463.

[8] Srinathkumar, S., "Eigenvalue/Eigenvector Assignment Using Output Feedback," IEEE Trans. on Automatic Control, Vol. 23, No. 1, January-February 1978, pp. 79-81.

[9] Maghami, P. G., and Juang, J-N., "Efficient Eigenvalue Assignment for Large Space Structures," AIAA Journal of Guidance, Control, and Dynamics, Vol. 13, No. 6, NovemberDecember 1990, pp. 1033-1039.

[10] Andry, A. N., Shapiro, E. Y., Chung, J. C., "Eigenstructure Assignment for Linear Systems," IEEE Trans. on Aerospace and Electronic Systems, Vol. 19, No. 5, SeptemberOctober 1983, pp. 711-729.

[11] Balas, M. J., "Some Trends in Large Space Structure Control Theory: Fondest Hopes; Wildest Dreams," IEEE Trans. on Automatic Control, Vol. AC-27, June 1982, pp. 522-535.

[12] Joshi, S. M., Control of Large Flexible Space Structures. edited by M. Thoma and A. Wyner, Volume 131, Lecture Notes in Control and Information Sciences, Springer-Verlag, Berlin, 1989. 
[13] Benhabib, R. J., Iwens, R. P., and Jackson, R. L., "Stability of Large Space Structure Control Systems Using Positivity Concepts," AIAA Journal of Guidance, Control, and Dynamics, Vol. 4, No. 5, September-October 1981, pp. 487-494.

[14] McLaren, M. D., and Slater, G. L., "Robust Multivariable Control of Large Space Structures Using Positivity," AIAA Journal of Guidance, Control, and Dynamics, Vol. 10, No. 4, July-August 1987, pp. 393-400.

[15] Desoer, C. A., and Vidyasagar, M., Feedback Systems: Input-Output Properties, Academic Press, New York, NY, 1975.

[16] Maghami, P. G., Gupta, S., and Joshi, S. M., "Design of Dissipative Low-Authority Controllers Using an Eigensystem Assignment Technique," Proceedings of the American Control Conference, Chicago, Illinois, June 1992, pp. 2780-2783.

[17] Maghami, P. G., and Gupta, S., "On Eigensystem Assignment With Dissipativity Constraints," Proceedings of the American Control Conference, San Francisco, CA, June 1993, pp. 1271-1276

[18] Laub, A. J., and Arnold, W. F., "Controllability and Observability Criteria for Multivariable Linear Second-Order Models," IEEE Trans. on Automatic Control, Vol. 29, No. 2, February 1984, pp. 163-165.

[19] Gardiner, J. D., "Stabilizing Control for Second-Order Models and Positive Real Systems," AIAA Journal of Guidance, Control, and Dynamics, Vol. 15, No. 1, January-February 1992, pp. 280-282.

[20] Varga, A., "A Schur Method for Pole Assignment," IEEE Trans. on Automatic Control, Vol. 26, No. 2, March-April 1981, pp. 517-519.

[21] Demmel, J. and Kagstrom, B.: "The Generalized Schur Decomposition of an Arbitrary Pencil $A-\lambda B$ : Robust Software with Error Bounds and Applications," Report UMINF — 170.90, University of Umea, Institute of Information Processing, Sweden, January 1990.

[22] Gupta, S., "Constrained Solution of a System of Matrix Equations," NASA Contractor Report No. 198288, Langley Research Center, Hampton, VA, February 1996. 


\begin{tabular}{|c|c|c|}
\hline Open-loop Eigenvalues & Damping Ratio & $\begin{array}{c}\text { Frequency } \\
\text { (rad/sec) }\end{array}$ \\
\hline$-0.0008 \pm 0.8180 j$ & 0.0010 & 0.8180 \\
$-0.0008 \pm 0.8301 j$ & 0.0010 & 0.8301 \\
$-0.0009 \pm 0.8565 j$ & 0.0010 & 0.8565 \\
$-0.0011 \pm 1.1308 j$ & 0.0010 & 1.1308 \\
$-0.0011 \pm 1.1401 j$ & 0.0010 & 1.1401 \\
$-0.0019 \pm 1.9100 j$ & 0.0010 & 1.9100 \\
$-0.0107 \pm 10.7278 j$ & 0.0010 & 10.7278 \\
$-0.0149 \pm 14.9425 j$ & 0.0010 & 14.9425 \\
$-0.0187 \pm 18.6919 j$ & 0.0010 & 18.6919 \\
$-0.0341 \pm 34.0618 j$ & 0.0010 & 34.0618 \\
\hline
\end{tabular}

Table 1. Open-loop eigenvalues

\begin{tabular}{|c|c|c|c|c|c|c|c|c|}
\hline $\begin{array}{c}\text { Mode } \\
\text { No }\end{array}$ & 1 & 2 & 3 & 4 & 5 & 6 & 7 & 8 \\
\hline 1 & 0.8315 & -0.0029 & 0.6053 & -0.0022 & -0.0346 & 0.3567 & -0.0347 & 0.6192 \\
2 & 0.0299 & 0.0059 & -0.0492 & -0.0002 & -0.6041 & -0.1195 & -0.6025 & -0.0040 \\
3 & 0.7498 & -0.0023 & -0.1425 & -0.0007 & 0.0614 & -0.9645 & 0.0611 & 0.2986 \\
4 & 0.0000 & -0.3740 & -0.0012 & 0.3563 & -0.0581 & 0.0030 & -0.1936 & 0.0113 \\
5 & -0.0014 & -1.0268 & 0.0030 & -0.4847 & -0.0434 & -0.0054 & -0.1433 & -0.0304 \\
6 & 0.1430 & 0.0067 & 0.3071 & 0.0047 & 0.0000 & -0.3702 & 0.0001 & -1.7897 \\
7 & -0.8114 & 0.0024 & 0.3292 & 0.0031 & 0.0010 & -0.5627 & -0.0013 & 1.3165 \\
8 & 0.0002 & -1.2687 & -0.0004 & 0.9737 & 0.3943 & 0.0019 & -0.9521 & -0.0072 \\
9 & 1.2653 & -0.0089 & -1.0289 & 0.0021 & -0.0009 & 0.3151 & 0.0164 & 0.3747 \\
10 & -0.0165 & 1.0266 & 0.0059 & 0.0330 & 0.6375 & 0.0238 & 0.6428 & 0.0114 \\
\hline \hline
\end{tabular}

Table 2. Modal Displacements at Control Stations. 


\begin{tabular}{|c|c|c|}
\hline Closed-loop Eigenvalues & Damping Ratio & $\begin{array}{c}\text { Frequency } \\
\text { (rad/sec) }\end{array}$ \\
\hline$-0.0818 \pm 0.8139 j$ & 0.1000 & 0.8180 \\
$-0.0036 \pm 0.8337 j$ & 0.0043 & 0.8338 \\
$-0.0133 \pm 0.8555 j$ & 0.0156 & 0.8556 \\
$-0.0352 \pm 1.1253 j$ & 0.0313 & 1.1258 \\
$-0.2343 \pm 1.1112 j$ & 0.2064 & 1.1356 \\
$-0.2059 \pm 1.9091 j$ & 0.1072 & 1.9202 \\
$-0.1326 \pm 10.7310 j$ & 0.0124 & 10.7319 \\
$-0.2305 \pm 14.9345 j$ & 0.0154 & 14.9363 \\
$-0.2526 \pm 18.6891 j$ & 0.0135 & 18.6908 \\
$-0.2818 \pm 34.0550 j$ & 0.0083 & 34.0561 \\
\hline \hline
\end{tabular}

Table 3. Closed-loop Eigenvalues With Gain, $G_{1}$.

\begin{tabular}{|c|c|c|}
\hline Closed-loop Eigenvalues & Damping Ratio & $\begin{array}{c}\text { Frequency } \\
\text { (rad/sec) }\end{array}$ \\
\hline$-0.0818 \pm 0.8139 j$ & 0.1000 & 0.8180 \\
$-0.0830 \pm 0.8259 j$ & 0.1000 & 0.8301 \\
$-0.0159 \pm 0.8540 j$ & 0.0186 & 0.8541 \\
$-0.1924 \pm 0.9840 j$ & 0.1919 & 1.0026 \\
$-0.3145 \pm 1.1213 j$ & 0.2701 & 1.1646 \\
$-0.7568 \pm 1.9581 j$ & 0.3605 & 2.0993 \\
$-0.2719 \pm 10.7216 j$ & 0.0254 & 10.7251 \\
$-1.5302 \pm 14.9152 j$ & 0.1021 & 14.9935 \\
$-0.6473 \pm 18.7686 j$ & 0.0345 & 18.7798 \\
$-0.4675 \pm 34.0439 j$ & 0.0137 & 34.0471 \\
\hline
\end{tabular}

Table 4. Closed-loop Eigenvalues With Gain, $G_{2}$. 


\begin{tabular}{|c|c|c|}
\hline Closed-loop Eigenvalues & Damping Ratio & $\begin{array}{c}\text { Frequency } \\
\text { (rad/sec) }\end{array}$ \\
\hline$-0.0818 \pm 0.8139 j$ & 0.1000 & 0.8180 \\
$-0.0830 \pm 0.8259 j$ & 0.1000 & 0.8301 \\
$-0.0857 \pm 0.8522 j$ & 0.1000 & 0.8565 \\
$-0.3648 \pm 1.0437 j$ & 0.3300 & 1.1056 \\
$-0.3463 \pm 1.1514 j$ & 0.2880 & 1.2024 \\
$-2.5906 \pm 1.5782$ & 1.2610 & 2.0544 \\
$-2.7128 \pm 9.5085 j$ & 0.2744 & 9.8879 \\
$-2.0568 \pm 14.5821 j$ & 0.1397 & 14.7265 \\
$-1.3549 \pm 18.5311 j$ & 0.0729 & 18.5806 \\
$-0.5327 \pm 34.0089 j$ & 0.0157 & 34.0131 \\
\hline \hline
\end{tabular}

Table 5. Closed-loop Eigenvalues With Gain, $G_{3}$.

\begin{tabular}{|c|c|c|}
\hline Closed-loop Eigenvalues & Damping Ratio & $\begin{array}{c}\text { Frequency } \\
\text { (rad/sec) }\end{array}$ \\
\hline$-0.0818 \pm 0.8139 j$ & 0.1000 & 0.8180 \\
$-0.0830 \pm 0.8259 j$ & 0.1000 & 0.8301 \\
$-0.0857 \pm 0.8522 j$ & 0.1000 & 0.8565 \\
$-0.3648 \pm 1.0436 j$ & 0.3300 & 1.1055 \\
$-0.3463 \pm 1.1514 j$ & 0.2881 & 1.2023 \\
$-2.6015 \pm 1.5893$ & 1.2631 & 2.0595 \\
$-2.8671 \pm 9.3545 j$ & 0.2930 & 9.7840 \\
$-3.2694 \pm 14.3800 j$ & 0.2217 & 14.7470 \\
$-1.8692 \pm 18.5982 j$ & 0.1000 & 18.6919 \\
$-0.6009 \pm 34.0359 j$ & 0.0177 & 34.0412 \\
\hline
\end{tabular}

Table 6. Closed-loop Eigenvalues With Gain, $G_{4}$. 


\begin{tabular}{|c|c|c|}
\hline Closed-loop Eigenvalues & Damping Ratio & $\begin{array}{c}\text { Frequency } \\
\text { (rad/sec) }\end{array}$ \\
\hline$-0.0818 \pm 0.8139 j$ & 0.1000 & 0.8180 \\
$-0.0046 \pm 0.8415 j$ & 0.0055 & 0.8415 \\
$-0.0072 \pm 1.1550 j$ & 0.0062 & 1.1550 \\
$-0.0081 \pm 1.2455 j$ & 0.0065 & 1.2455 \\
$-0.0997 \pm 1.4382 j$ & 0.0692 & 1.4417 \\
$-0.1580 \pm 2.0261 j$ & 0.0778 & 2.0322 \\
$-0.0461 \pm 10.8179 j$ & 0.0043 & 10.8180 \\
$-0.1222 \pm 14.9897 j$ & 0.0081 & 14.9902 \\
$-0.0696 \pm 18.7224 j$ & 0.0037 & 18.7225 \\
$-0.0599 \pm 34.0745 j$ & 0.0018 & 34.0745 \\
\hline \hline
\end{tabular}

Table 7. Closed-loop Eigenvalues With Position Gain, $G_{p 1}$ and Rate Gain $G_{r 1}$.

\begin{tabular}{|c|c|c|}
\hline Closed-loop Eigenvalues & Damping Ratio & $\begin{array}{c}\text { Frequency } \\
\text { (rad/sec) }\end{array}$ \\
\hline$-0.0818 \pm 0.8139 j$ & 0.1000 & 0.8180 \\
$-0.0830 \pm 0.8259 j$ & 0.1000 & 0.8301 \\
$-0.0857 \pm 0.8522 j$ & 0.1000 & 0.8565 \\
$-0.1575 \pm 1.3598 j$ & 0.1151 & 1.3689 \\
$-0.1682 \pm 1.6559 j$ & 0.1010 & 1.6644 \\
$-0.5009 \pm 3.7347 j$ & 0.1329 & 3.7682 \\
$-1.0728 \pm 10.6741 j$ & 0.1000 & 10.7278 \\
$-1.2597 \pm 18.5179 j$ & 0.0679 & 18.5607 \\
$-3.5796 \pm 20.9683 j$ & 0.1683 & 21.2717 \\
$-0.7608 \pm 34.7506 j$ & 0.0219 & 34.7589 \\
\hline
\end{tabular}

Table 8. Closed-loop Eigenvalues With Position Gain, $G_{p 4}$ and Rate Gain $G_{r 4}$. 


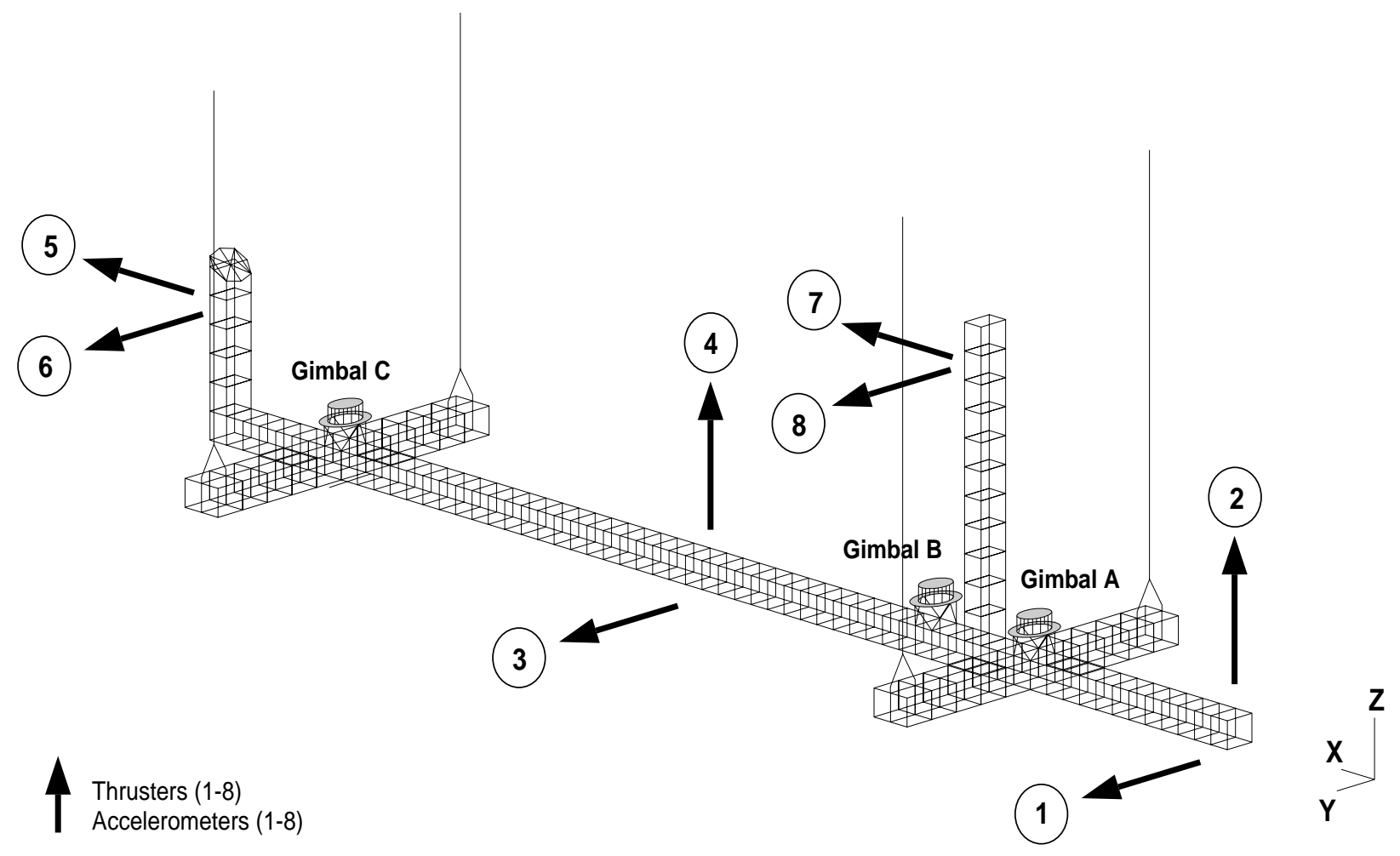

Figure 1. Schematic of Phase 2 CEM Structure, With Location of 8 Control Stations. 